\title{
Jonas Scherner
}

\section{Europas Beitrag zu Hitlers Krieg}

\author{
Die Verlagerung von Industrieaufträgen der Wehrmacht in die \\ besetzten Gebiete und ihre Bedeutung für die deutsche Rüstung im \\ Zweiten Weltkrieg
}

\section{Einleitung}

In der Geschichtsschreibung zum Zweiten Weltkrieg ist in jüngster Zeit die Bedeutung des besetzten Europa für die deutsche Kriegsführung in den Vordergrund gerückt. Rolf-Dieter Müller zeigte, dass der ausländische militärische Beitrag für die deutsche Ostfront beachtlich war ${ }^{1}$. Hiernach war dort auf dem Höhepunkt des Zweiten Weltkriegs etwa jeder dritte Soldat auf deutscher Seite ein Ausländer. Schon länger bekannt ist, dass das Reich in finanzieller Hinsicht beträchtlich von den besetzten Gebieten profitierte, was sich an der Summe der von Deutschland erzwungenen Clearingkreditvergabe durch die besetzten Länder und den ihnen auferlegten Besatzungskosten ermessen lässt, die etwa $100 \mathrm{Mrd}$. RM betragen haben dürften ${ }^{2}$. Unscharf bleibt aber bis heute, wofür die aus den besetzten Ländern herausgepressten Mittel verwendet wurden. Adam Tooze vermutet, dass der mit Abstand wichtigste Beitrag der besetzten Gebiete der Einsatz der Zwangsarbeiter für die deutsche Kriegs- und damit auch Rüstungsproduktion gewesen sei ${ }^{3}$. Richard Overy hingegen glaubt, dass die Güter und Dienstleistungen, die die Wehrmacht in den besetzten Ländern nachfragte, den größten Anteil an deren Inanspruchnahme für die deutsche Kriegswirtschaft gehabt haben könnten, nicht ohne hervorzuheben, dass diese Frage noch nicht systematisch untersucht worden sei ${ }^{4}$. Keine Rolle spielte bis vor kurzem in der Literatur die eigentlich naheliegende

\footnotetext{
1 Rolf-Dieter Müller, An der Seite der Wehrmacht. Hitlers ausländische Helfer beim „Kreuzzug gegen den Bolschewismus" 1941-1945 (Berlin 2007) 243f.

2 Jonas Scherner, Der deutsche Importboom während des Zweiten Weltkriegs. Neue Ergebnisse zur Struktur der Ausbeutung des besetzten Europas auf der Grundlage einer Neuschätzung der deutschen Handelsbilanz, in: Historische Zeitschrift (im Erscheinen).

3 Adam Tooze, The Wages of Destruction. The Making and Breaking of the Nazi Economy (London 2006) 410, 640.

${ }^{4}$ Richard J. Overy, The Economy of the German „New Order", in: ders., Gerbard Otto, Johannes Hourwink ten Cate (Hrsg.), Die „Neuordnung“ Europas. NS-Wirtschaftspolitik in den besetzten Gebieten (Berlin 1997) 11-28, hier: 17.
} 
Hypothese, dass das Reich die gewaltigen, ihm aus den besetzten Gebieten zufließenden Gelder auch zur Finanzierung größerer deutscher Überschüsse im Warenhandel verwendet haben könnte. Der Grund dafür, warum dieser Gedanke nicht geäußert wurde, ist einfach: Nach der deutschen Außenhandelsstatistik hatte das Reich während der Kriegsjahre den besetzten Gebieten gegenüber gerade einmal ein kumuliertes Handelsbilanzdefizit von ca. 5 Mrd. RM - was also nur einem Bruchteil der von den besetzten Ländern empfangenen finanziellen Mittel entspricht. Allerdings ist die deutsche Importstatistik, im Unterschied zur Ausfuhrstatistik, unter anderem infolge von kriegsbedingten Erhebungsproblemen massiv verzerrt $^{5}$. Obwohl sich die Behörden über die mangelnde Aussagekraft der Einfuhrstatistik durchaus im Klaren waren, veröffentlichten sie aus propagandistischen Gründen diese Zahlen im Sommer 1944, konnte doch so dem Ausland gegenüber suggeriert werden, Deutschland beute das besetzte Europa nicht aus. Tatsächlich kam es aber zu hohen Importüberschüssen des Reichs mit den besetzten Gebieten, die sich nach bisherigen Erkenntnissen auf mindestens $42 \mathrm{Mrd}$. RM beziffern lassen.

Doch wer war der größte Nutznießer dieser Einfuhren? Waren es die deutschen Konsumenten oder etwa die Wehrmacht? Dass letztere davon profitierte, scheint klar zu sein ${ }^{6}$; es kam nämlich zu, im übrigen völkerrechtswidrigen ${ }^{7}$, Verlagerungen von Rüstungsaufträgen an die Industrie der besetzten Gebiete. Die exakte Größe dieser militärischen Auftragsverlagerung und damit auch ihr Anteil an der Einfuhr des Reiches aus den besetzten Gebieten sind allerdings nach wie vor unbekannt.

Genauere Kenntnisse über die militärische Auftragsverlagerung können zu zwei zentralen Debatten über die deutsche Kriegswirtschaft beitragen. Bei der ersten dieser Debatten handelt es sich um die sogenannte Blitzkriegshypothese, die 1945 im Abschlussbericht des United States Strategic Bombing Survey, einer von einer amerikanischen Untersuchungskommission über die Auswirkungen des alliierten Bombenkriegs auf die deutsche Kriegswirtschaft verfassten Arbeit, formuliert und später u.a. von dem britischen Wirtschaftshistoriker Alan Milward weiter ausgebaut wurde ${ }^{8}$. Nach dieser Hypothese hat Deutschland erst mit der Niederlage vor Moskau und dem Kriegseintritt der USA seine Wirtschaft konsequent auf einen lang andauernden Krieg umgestellt. Fast alle Argumente der Blitzkriegshypothese sind in den letzten zwanzig Jahren jedoch widerlegt worden - nämlich das angeb-

\footnotetext{
${ }^{5}$ Für das Folgende, vgl. Scherner, Der deutsche Importboom.

${ }^{6}$ Jonas Scherner, Jochen Streb, Das Ende eines Mythos? Albert Speer und das so genannte Rüstungswunder, in: Vierteljahrschrift für Sozial- und Wirtschaftsgeschichte 93 (2006) 172-196, hier: $179 f$.

7 Vgl. z.B. Ludwig Nestler, Europa unterm Hakenkreuz. Die faschistische Okkupationspolitik in Belgien, Luxemburg und den Niederlanden (1940-1945) (Berlin 1990) 41.

8 United States Strategic Bombing Survey (Hrsg.), The Effects of Strategic Bombing on the German War Economy. Overall Economic Effects Division, October 31, 1945, 1 (New York 1976) 6; Burton H. Klein, Germany's Economic Preparations for War (Cambridge/Mass. 1959); Alan S. Milward, Die deutsche Kriegswirtschaft 1939-1945 (Stuttgart 1966).
} 
lich hohe Konsumniveau in Deutschland in den ersten beiden Kriegsjahren ${ }^{9}$, dass der Frankreichfeldzug als Blitzkrieg geplant war ${ }^{10}$ sowie der vermeintlich kaum erfolgte Ausbau einer industriellen Basis für einen langen Krieg ${ }^{11}$. Nicht widerlegt ist allerdings das auch noch in jüngster Zeit in der Literatur aufzufindende Argument Milwards, dass es erst nach 1941, nachdem dem Rüstungsministerium die entsprechenden Zuständigkeiten übertragen worden waren, zu einer verstärkten Ausbeutung der besetzten Gebiete gekommen sei: „The failure of the Blitzkrieg meant that the only solution possible was an European one (...). The end of the Blitzkrieg meant the end of the self-imposed limitations on German economic policy abroad." 12 Diese Sicht der Dinge stützt sich unter anderem auf Aussagen, die der im Februar 1942 zum Rüstungsminister ernannte Albert Speer in einem Verhör unmittelbar nach Kriegsende machte ${ }^{13}$. Allerdings haben neuere Forschungen darauf hingewiesen, dass Nachkriegsaussagen Albert Speers nicht nur, was sein Wissen um die Verbrechen des NS-Regimes, sondern auch was sein Handeln in der kriegswirtschaftlichen Sphäre anbelangt, nicht in jedem Fall für bare Münze genommen werden sollten, lag doch Speer (und auch seinen Mitarbeitern) daran, sich als „unpolitische“ Technokraten zu gerieren, die sich lediglich darum bemüht hätten, die ihnen übertragenen rüstungswirtschaftlichen Probleme effizient zu lö$\operatorname{sen}^{14}$.

Damit rückt eine zweite Debatte in das Blickfeld, zu der das Wissen um die militärische Auftragsverlagerung beitragen kann, nämlich die in jüngster Zeit entbrannte Diskussion um das so genannte Rüstungswunder. Nach der neueren Literatur ist es, anders als Speer und seine Mitarbeiter behaupteten, zunehmend fragwürdig geworden, dass der seit Anfang $1942 \mathrm{zu}$ beobachtende massive Anstieg der Rüstungsproduktion großenteils auf unter dem neuen Rüstungsminister eingeführte Rationalisierungsmaßnahmen zurückzuführen ist. Vielmehr wird er als eine mehr oder weniger zwangsläufige Entwicklung betrachtet, für welche die Grundlagen bereits in der Zeit zuvor gelegt worden sind ${ }^{15}$. In diesem Zusammen-

9 Richard J. Overy, War and Economy in the Third Reich (Oxford 1994) 278, 288-90. Vgl. auch W. Abelshauser, Germany: Guns, Butter and Economic Miracles, in: Harrison, M. (Hrsg.), The Economics of World War II: Six Great Powers in International Comparison (Cambridge 1998) 122-76, hier: 151-153.

${ }^{10}$ Karl-Heinz Frieser, Blitzkrieg-Legende. Der Westfeldzug 1940 (München ${ }^{32005) . ~}$

11 Jonas Scherner, Nazi Germany's Preparation for War. Evidence from Revised Industrial Investment Series, in: European Review of Economic History 14 (2010) 433-468.

12 Alan S. Milward, The New Order and the French Economy (Oxford 1970) 41f. Vgl. auch Milward, Die deutsche Kriegswirtschaft 79, 109; Harald Winkel, Die Ausbeutung des besetzten Frankreichs, in: Kriegswirtschaft und Rüstung 1939-1945, hrsg. v. Friedrich Forstmeier, HansErich Volkmann (Düsseldorf 1977) 373; Werner Röhr, Forschungsprobleme zur deutschen Okkupationspolitik im Spiegel der Reihe „Europa unterm Hakenkreuz“, in: Europa unterm Hakenkreuz. Die Okkupationspolitik des deutschen Faschismus (1938-1945) 8, Analysen, Quellen, Register, hrsg. v. Wolfgang Schumann u. a. (Heidelberg 1996) 25-343, hier: 242; Richard J. Overy, The Economy of the German „New Order“ 17.

13 Milward, The New Order 121.

14 Scherner, Streb, Das Ende eines Mythos; Tooze, The Wages of Destruction.

15 Scherner, Streb, Das Ende eines Mythos; Tooze, The Wages of Destruction. 
hang stellt sich natürlich die Frage, in welchem Maß die militärische Auftragsverlagerung einen Beitrag zum Rüstungswunder leistete und wann ihre Anfänge zu datieren sind.

Im Folgenden stehen der zeitliche Verlauf der militärischen Auftragsverlagerung und ihr quantitativer Umfang im Mittelpunkt der Betrachtung. Darüber hinaus sollen aber weitere Fragen beantwortet werden, nämlich: Wie hoch war der Anteil der einzelnen besetzten Länder an der Verlagerungsproduktion, und inwieweit kam es bei diesen zu einer Spezialisierung auf bestimmte von der Wehrmacht nachgefragte industrielle Güter? Sind zeitliche Zäsuren festzustellen, und worauf sind sie gegebenenfalls zurückzuführen? Lässt sich eine Intensivierung der militärischen Auftragsverlagerung nachweisen, nachdem Speers Ministerium die entsprechenden Zuständigkeiten übertragen worden waren? Wie wurde die militärische Auftragsverlagerung abgewickelt, und welchen Anteil hatte sie an den deutschen Importen? Gab es limitierende Faktoren?

Im ersten Abschnitt werden die Genese und der verwaltungsmäßige Ablauf der militärischen Auftragsverlagerung skizziert. Anschließend wird versucht, die militärische Auftragsverlagerung zu quantifizieren und Aussagen über ihre Bedeutung für die deutsche Rüstungswirtschaft zu machen. Vor diesem Hintergrund sollen dann im dritten Abschnitt die weiteren aufgeworfenen Fragen beantwortet werden.

\section{Die administrative Entwicklung der militärischen Auftragsverlagerung}

Zunächst soll der Begriff der militärischen industriellen Auftragsverlagerung genauer definiert werden. Zu unterscheiden ist er nämlich von dem bis Ende 1941 im zollrechtlichen Sprachgebrauch verwendeten Begriff der Verlagerung von „Rüstungsgut"16. Als Rüstungsgut wurden bis zu diesem Zeitpunkt zollrechtlich nämlich nicht nur Rüstungsgüter im eigentlichen Sinne, wie Waffen, und Vorleistungen für ihre Herstellung, sondern auch so genannte „Entlastungsaufträge“, also Aufträge, die deutsche Rüstungsfirmen von der zivilen Produktion entlasteten, bezeichnet ${ }^{17}$. Wenn aber im Folgenden von der militärischen Auftragsverlagerung bzw. von „Rüstungsgütern“ die Rede ist, so sind nur industrielle Fertigwaren gemeint, deren Nachfrager mittelbar oder unmittelbar die Wehrmacht war und die - nach einer weiteren zeitgenössischen Definition - aufgrund „direkter oder indirekter Wehrmachtaufträge“ im Ausland gefertigt wurden ${ }^{18}$. Der hier ver-

16 Bundesarchiv-Militärarchiv (im Folgenden BA-MA) RW 19/1603, Der Reichsfinanzminister, Erlass vom 31. August 1940, Bl.19; Bundesarchiv Berlin (im Folgenden BArch) R 2/ 58993, Reichsfinanzministerium an den Oberfinanzpräsident Thüringen, Zollbehandlung des Warenverkehrs bei der Verlagerung kriegswichtiger Aufträge (Verlagerungsgut), 5. Oktober 1941, Bl. 197.

17 BA-MA RW 19/1614, Reichsfinanzministerium an OKW, 7.11.1941, Bl.27.

18 Vgl. BArch R 2/58993, Verzollung bei Auftragsverlagerung von Wehrmachtaufträgen in Frankreich, Mai 1941, Bl.109. Ein indirekter Wehrmachtauftrag lag z.B. vor, wenn die Luftwaffe 
wendete Begriff der Fertigwaren entspricht dabei der Klassifikation der deutschen Außenhandelsstatistik, umfasst also End- und Vorerzeugnisse, nicht aber Halbwaren. Weiterhin ist einschränkend zu bemerken, dass Marktkäufe der Wehrmacht im Ausland, also der Erwerb von Gütern, denen kein Fertigungsauftrag zugrunde lag, nicht unter den Begriff der Auftragsverlagerung fielen. Und schließlich handelte es sich nicht um solche Güter, die vor Ort durch die jeweiligen deutschen Besatzungstruppen konsumiert oder genutzt wurden.

Im allgemeinen wurde unmittelbar nach der Besetzung die Industrie der jeweiligen Länder für die militärische Auftragsverlagerung in Anspruch genommen. Das war insbesondere im Protektorat der Fall ${ }^{19}$. Die Böhmische Waffenfabrik AG in Prag hatte nach eigener Aussage sogar schon unmittelbar nach dem Münchner Abkommen der Wehrmacht über sudetendeutsche Mittelsmänner ihre Dienste angeboten $^{20}$. Die rüstungswirtschaftliche Ausbeutung der besetzten westlichen Gebiete war ebenfalls bereits kurz nach ihrer Eroberung eingeleitet worden ${ }^{21}$. Zum Teil gingen die Planungen für die Einbindung dieser Länder in die deutsche Rüstungsproduktion noch auf die Zeit unmittelbar vor der Besetzung zurück ${ }^{22}$. Angesichts dieser frühzeitigen Absichten ist es dann auch nicht überraschend, dass z. B. bereits seit Ende Juli 1940 auftragsverlagerte Waren aus den besetzten Westgebieten zollfrei ins Reich eingeführt werden konnten ${ }^{23}$. In den letztgenannten Gebieten - nicht anders als im Protektorat - hatte die einheimische Industrie ein Interesse an deutschen Aufträgen, um ihr Überleben zu sichern ${ }^{24}$. Im Generalgouvernement, in dem zunächst die Demontage von Industriebetrieben im Vordergrund gestanden hatte, setzte sich seit Herbst 1940 auf breiter Front die bereits seit dem Vorjahr vom Wehrwirtschafts- und Rüstungsamt geforderte und in

bei Junkers Flugzeuge bestellt hatte und Junkers die dafür benötigten Tragflächen von einem ausländischen Produzenten fertigen ließ.

19 BA-MA RL 3/653, LC 6/II, Besichtigung Böhmisch-Mährischer Waffenfirmen vom 28.3.1.4.1939, 6.4.1939.

20 BA-MA RL 3/653, Böhmische Waffenfabrik AG, Prag an Oberstleutnant Wendt, Verbindungsoffizier des Oberkommandos der Wehrmacht/W Stb/bei XIII Armeekorps vom 21.3.1939. Das scheint kein Einzelfall gewesen zu sein, vgl. Alice Teichova, Robert Waller, Der tschechoslowakische Unternehmer am Vorabend und zu Beginn des Zweiten Weltkriegs, in: Zweiter Weltkrieg und sozialer Wandel. Achsenmächte und besetzte Gebiete, hrsg. v. Wactaw Dtugoborski (Göttingen 1981) 288-302, hier: 299-302.

${ }^{21}$ Vgl. z. B. Politisches Archiv des Auswärtigen Amts (im Folgenden PA AA) R 105284, Hitler an Göring, 16.5.1940; BA-MA RL 3/1613, Ausnutzung der Industrie in den besetzten Westgebieten für die deutsche Luftwaffenrüstung, Stand 31.12.1940; BA-MA RL 3/1646, Generalluftzeugmeister, Betr.: Inanspruchnahme Dänemarks und Norwegens für Zwecke der deutschen Luftwaffenrüstung, 9.5.1940; Georg Thomas, Geschichte der deutschen Wehr- und Rüstungswirtschaft (1918-1943/45) (Boppard 1966) 209.

22 Vgl. z. B. im Fall Dänemarks und Norwegens, PA AA R 105284, OKW an Auswärtiges Amt, 2.4.1940.

23 BArch R 2/58993, Der Reichsfinanzminister, Zollerlaß für Rüstungsaufträge aus den besetzten Westgebieten, 31. Juli 1940, Bl.24.

${ }^{24}$ Vgl. z. B. BA-MA RW 25/116, ZAST Belgien und Nordfrankreich, Tätigkeitsbericht Januar 1941, Bl.6. 
Einzelfällen ${ }^{25}$ schon verfolgte Linie durch, die wider Erwarten gut eingerichtete polnische Industrie für die deutsche Rüstungsproduktion zu nutzen, zumal ihr Standort gerade unter dem Aspekt der Luftsicherheit Vorteile bot ${ }^{26}$. Die größtmögliche Einbindung aller besetzten Gebiete in die deutsche Kriegswirtschaft im Allgemeinen und in die Rüstungsproduktion im Speziellen, die in Ansätzen durch die rüstungswirtschaftlichen Instanzen aller seit 1939 besetzten Gebiete in Angriff genommen worden war, erklärte schließlich ein Erlass Görings vom Spätsommer 1940 zur generellen wirtschaftspolitischen Leitlinie ${ }^{27}$.

Allerdings betonte Göring kurze Zeit später, dass die Verlagerung ziviler Güter Vorrang haben sollte, weil komplexe Rüstungsgüter eine längere Anlaufzeit benötigten. Außerdem befürchtete man bei der Produktion von Rüstungsgütern in besetzten Gebieten eher Sabotageakte, eine erhöhte Gefährdung der Geheimhaltung technischer Details von Kriegsgerät und eine geringe Motivation der Beschäftigten $^{28}$. Daher sollten seit Herbst 1940 zum Zweck der Produktionssteigerung von Rüstungsgut Unternehmen im Reich von der gesamten verlagerungsfähigen zivilwirtschaftlichen Fertigung entlastet werden und deren Auslagerung „im größtmöglichen Umfang“ durchgeführt werden ${ }^{29}$. Dabei waren generell lediglich Betriebe in den besetzten Gebieten heranzuziehen, die die Produktion von auftragsverlagerten Gütern in einem nennenswerten Umfang ohne Investitionen aufnehmen konnten ${ }^{30}$. Zudem sollte es sich aus Effizienzgründen nur um solche Unternehmen handeln, die bereits Erfahrung in der Produktion von Gütern hatten, die denen der verlagerten Ware ähnlich waren. Wenn auch die zivilwirtschaftliche Verlagerung im Vordergrund stehen sollte, ging man in der Praxis dann doch,

25 Vgl. z.B. BArch 8135/7828, Prüfungsbericht der Deutschen Revisions- und Treuhand AG über den Jahresabschluß 1942 der „Pionier“ Werkzeugmaschinenfabrik GmbH, Warschau. Dieser Betrieb wurde bereits im Oktober 1939 von der Wehrwirtschaftsstelle Lodz „sichergestellt“, um ihn vor der Ausplünderung zu schützen. Am 18.3.1940 wurde er zum Wehrmachtbetrieb erklärt und unterstand später der Verwaltungsgesellschaft für heereseigene Betriebe, der MONTAN GmbH. Zur MONTAN, vgl. Barbara Hopmann, Von der Montan zur Industrieverwaltungsgesellschaft (IVG) 1916-1951 (Stuttgart 1996). Im März 1940 waren bereits 3000 Arbeitskräfte in drei Werken für die deutsche Luftwaffenrüstung tätig. BA-MA RL 3/2638, Gefolgschaftsbewegung der gesamten statistisch erfassbaren Luftwaffenindustrie.

26 Thomas, Geschichte der deutschen Wehr- und Rüstungswirtschaft $164 \mathrm{f}$.

27 BA-MA RW 35/774, Reichsmarschall Göring, Planmäßige Ausnutzung der Wirtschaft der besetzten Gebiete für die deutsche Kriegswirtschaft, 26.8.1940.

28 BA-MA RW 35/774, Göring an den Reichswirtschaftsminister, 7.10.1940, Betr. Auftragsverlagerung an die Industrie in den bes. Westgebieten; Overy, The Economy of the German „New Order" 16.

29 BA-MA RW 35/774, Reichswirtschaftsminister an den Mobbeauftragten der Wirtschaftsgruppe der Eisen und Metall verarbeitenden Industrie in den besetzten Gebieten.

30 BArch R 3101/32257, Reichswirtschaftsminister an die Mobbeauftragten der Wirtschaftsgruppen der Eisen und Metall verarbeitenden Industrie, 18.10.1940, Bl.21. Zum Prozedere der Auftragsverlagerung und zur Feststellung der Verlagerungswürdigkeit, vgl. ebd. sowie BArch $\mathrm{R}$ 2/265, OKW und Reichswirtschaftsminister, Ausführungsbestimmungen zu der Anordnung des Reichsmarschalls des Grossdeutschen Reiches über planmässige Ausnutzung der Wirtschaft der besetzten westlichen Gebiete für die deutsche Kriegswirtschaft vom 28.8.1940 - V.P. 14395, 30.4. 1941, Bl.145-147. 
wie an späterer Stelle gezeigt wird, dazu über, in den besetzten Gebieten in zunehmendem Maß Rüstungsgüter fertigen zu lassen. Wohl um die erwähnten Probleme der Anlaufzeit und der Geheimhaltungsgefährdung zu entschärfen, wurden aber in großem Umfang Teile und nicht Endprodukte hergestellt.

Die Auftragsvergabe in die westlichen Besatzungsgebiete wurde über in den jeweiligen Ländern neu eingerichtete Zentralauftragsstellen (ZAST) gelenkt, wobei Aufträge für die Wehrmacht Vorrang hatten ${ }^{31}$. Die Möglichkeit der Erfüllung größerer Aufträge wurde - gerade was die Verfügbarkeit der notwendigen Rohstoffe anbelangt - durch die ZAST geprüft. Die ZAST erfasste keine Rohstoff-, Forstund Ernährungskäufe und generell nur Aufträge, die ein Volumen von mehr als 5000 RM hatten ${ }^{32}$. Allerdings erfolgte die Auftragsverlagerung auch in den westlichen Besatzungsgebieten nicht immer über die ZAST ${ }^{33}$. Im Protektorat und im Generalgouvernement dagegen gab es keine ZAST, was wohl auf den unterschiedlichen Charakter der Besatzungsregimes in diesen Ländern im Vergleich zu den west- und nordeuropäischen Staaten zurückzuführen war, die sich ja bereits in ihren Bezeichnungen zeigte ${ }^{34}$.

Im Laufe des Jahres 1942 wurden die Zentralauftragsstellen dem Reichsministerium für Bewaffnung und Munition unterstellt, dem im Frühjahr 1943 praktisch die gesamte Verantwortung für die Rüstungsproduktion in den besetzten Gebieten übertragen wurde ${ }^{35}$. Von diesem Zeitpunkt an sollte die Auftragsvergabe an die ZAST nur noch entweder durch von Ausschüssen und Ringen damit betraute Firmen oder direkt durch militärische Dienststellen erfolgen ${ }^{36}$. Mit einem Erlass Speers vom Dezember 1943 wurde eine weitere Zentralisierung der Auftragsverlagerung in die besetzten Gebiete angestrebt. Denn jetzt sollten nur noch die Ringe und Ausschüsse, nicht aber mehr die Wehrmachtsstellen als

31 BA-MA RW 35/774, Reichsmarschall Göring, Planmäßige Ausnutzung der Wirtschaft der besetzten Gebiete für die deutsche Kriegswirtschaft, 26.8.1940; BArch R 3/3029, Deutsche Aufträge nach Frankreich, herausgegeben von der Zentralauftragsstelle Frankreich, 1.10.1943.

32 BArch R 3/3029, Bl. 11.

33 Teilweise beauftragten die Wehrmacht oder Unternehmen direkt ausländische Firmen. Vgl. z.B. BArch R 3101/32261, Deutsche Wirtschaftspolitik in Frankreich, 10.7.1943, Bl.3; BA-MA RW 25/116, ZAST Belgien und Nordfrankreich, Tätigkeitsbericht Januar 1941, Bl. 8-10.

34 Vgl. z. B. für das Protektorat, BArch R 3/504, Besprechungsnotiz, 30.1.1943. So sprach die Forschungsstelle für Wehrwirtschaft von dem Protektorat als einer faktischen „Provinz“ und vom Generalgouvernement als einem „Nebenland“ des Reichs. Christoph Buchbeim, Die besetzten Länder im Dienste der deutschen Kriegswirtschaft während des Zweiten Weltkriegs. Ein Bericht der Forschungsstelle für Wehrwirtschaft, in: Vierteljahrshefte für Zeitgeschichte 34 (1986) 117-145, hier 126f. Zur rüstungswirtschaftlichen Organisation in den Westgebieten, vgl. z. B. Hans Umbreit, Der Militärbefehlshaber in Frankreich 1940-1944 (Boppard 1968) 34-36.

35 BA-MA RW 35/774, Aktenvermerk 25.7.1942; Bernd Zielinski, Die deutsche Arbeitseinsatzpolitik in Frankreich 1940-1944, in: Overy, Otto, Houwink ten Cate, „Neuordnung“ 109-132, hier: 124.

${ }^{36}$ BArch R 3101/32261, Einschaltung der Ausschüsse und Ringe bei der rüstungswirtschaftlichen Nutzbarmachung Frankreichs, 1.6.1943, Bl.23ff. 
direkte Auftraggeber fungieren ${ }^{37}$. Überwachungsinstanz wurde das neu geschaffene Planungsamt im Rüstungsministerium ${ }^{38}$. Mit der Einbindung von Ringen und Ausschüssen ist somit, allerdings mit einiger zeitlicher Verzögerung, das so genannte Prinzip der Selbstverantwortung der Industrie vollständig auf die besetzten Gebiete übertragen worden ${ }^{39}$, wenn es auch in der Praxis nicht immer konsequent eingehalten wurde ${ }^{40}$. Mithilfe von Patenfirmen sollte die Produktivität ausländischer Firmen, an die Aufträge gegeben wurden, gesteigert werden. Eine Patenfirma war ein ausgewählter deutscher Rüstungsproduzent, der ein oder mehrere ausländische Unternehmen beriet. Diese Unterstützung sollte u. a. bei der Arbeitsvorbereitung und bei der Rationalisierung - durch technischen Erfahrungsaustausch - erfolgen ${ }^{41}$. Daneben gab es noch die Institution der Leitfirmen, die sämtliche Unternehmen eines bestimmten Fertigungsbereichs betreuten. Hauptausschüsse und Ringe benannten darüber hinaus Länderbeauftragte, welche die in ihrem Bereich vorhandenen Kapazitäten zu ermitteln und Produktionspläne auszuarbeiten hatten, wobei Größenvorteile auszuschöpfen waren ${ }^{42}$.

An der im Laufe des Jahres 1940 eingeleiteten Politik - weitgehende Ausnutzung der besetzten Gebiete, Fertigung standardisierter Rüstungsgüter bzw. von Teilen und zivile Produktionen - hielt im Grundsatz auch das Rüstungsministerium fest, nachdem es die Kompetenzen über die Auftragsverlagerung erhalten hatte. Ja, es versuchte Ende 1943 aus Gründen der Risikostreuung und der Luftsicherheit sogar eine Forcierung der Auftragsverlagerung zu erreichen, da Unternehmen in den besetzten Gebiete in einem geringeren Maß als die Produktionsstätten im Reich Luftangriffen ausgesetzt waren ${ }^{43}$. Dazu wurde im Fall Frankreichs das nach dem französischen Industrie- und dem deutschen Rüstungsminister benannte Bichelonne-Speer-Abkommen geschlossen. Dort sagte die deutsche Seite einen weitgehenden Stopp der Zwangsrekrutierung französischer Arbeitskräfte für den Reichseinsatz zu, falls im Gegenzug die industrielle Produktion Frankreichs für deutsche Zwecke, insbesondere Konsumgüter und Vorleistungen für

37 BArch R 8 IX/19, Erlass über Auftragsverlagerung in die besetzten Westgebiete des Reichsministeriums für Rüstung und Kriegsproduktion, 3.12.1943.

38 BArch R 3/470, Planungsamt, Aktenvermerk, 22.12.1943.

39 Milward, The New Order 138-140.

40 BArch R 8 IX/19, ZAST Frankreich an Reichsstelle für technische Erzeugnisse, 19.3.1944.

${ }^{41}$ Vgl. z.B. BArch R 3/3152, Rüstungsobmann in Frankreich an Carl Zeiss Jena, 30.9.1943, Bl.2-5. Ähnliches galt auch im Fall der Auftragsverlagerung in verbündete Länder. Vgl. z.B. BArch R 3/1645, Ungarische Wirtschaftseindrücke, 27.5.-3.7.1944, Bl.140.

42 BArch R 3101/32261, Einschaltung der Ausschüsse und Ringe bei der rüstungswirtschaftlichen Nutzbarmachung Frankreichs, 1.6.1943, Bl.26f.

${ }^{43}$ BA-MA RW 35/774, Reichsminister für Rüstung und Kriegsproduktion, Erlass über Auftragsverlagerung in die besetzten Westgebiete. Vom 3. Dezember 1943; BArch R 3/470, Planungsamt, Aktenvermerk, 22.12.1943; Hans Umbreit, Die deutsche Herrschaft in den besetzten Gebieten 1942-1945, in: Militärgeschichtliches Forschungsamt (Hrsg.), Das Deutsche Reich und der Zweite Weltkrieg 5/2 (Stuttgart 1999) 3-274, hier: 188. 
Kriegsgerät, gesteigert würde ${ }^{44}$. Angesichts der stagnierenden französischen Verlagerungsproduktion wurde insbesondere entschieden, dass der Abzug von Arbeitskräften aus rüstungswichtigen Unternehmen gestoppt werden sollte ${ }^{45}$. Kriegswichtige Unternehmen in den westlichen Besatzungsgebieten einschließlich Italiens wurden zu so genannten S(perr)-betrieben erklärt ${ }^{46}$. Zum gleichen Zweck der Steigerung der Verlagerungsproduktion wurde Anfang 1944 das im März 1943 auch für die besetzten Gebiete eingeführte, bis dahin grundsätzlich nur für die Konsumgütereinfuhr geltende Preisausgleichsverfahren generell auf den Import von Rohstoffen, Halb- und Fertigwaren aus den Westgebieten ausgedehnt ${ }^{47}$. Das Preisausgleichsverfahren führte zu einer Verbilligung der eingeführten Waren. Ziel dieses Verfahrens war, die Preise im Reich für die privaten Verbraucher konstant zu halten. Dementsprechend waren Lieferungen an öffentliche Bedarfsträger, also auch an die Wehrmacht, von dem Preisausgleich seit seiner Einführung ausgenommen $^{48}$. Dabei blieb es auch im Folgenden ${ }^{49}$. Allerdings wurden seit Februar 1944 Zulieferungen und Zubehör für Kriegsgerät doch in einem beschränkten Umfang in den Preisausgleich einbezogen ${ }^{50}$. Im Fall der besetzten Gebiete kam neben der Preisniveaustabilität für private Verbraucher noch eine zweite Zielsetzung hinzu, die durch den Preisausgleich erreicht werden sollte, denn man wollte die stark zunehmende deutsche Clearingverschuldung mit diesen Ländern eindämmen. Da-

${ }^{44}$ Bernd Zielinski, Die deutsche Arbeitseinsatzpolitik in Frankreich 1940-1944 126f; BArch R 3/470, Anweisung des Rüstungsministers an alle Ausschüsse und Ringe und mit der Produktionssteigerung beauftragten Gruppen, Betr.: Kriegswirtschaftliche Nutzbarmachung der besetzten Westgebiete, 14.12.1943; Umbreit, Die deutsche Herrschaft 188.

45 BA-MA RW 35/774, Reichsminister für Rüstung und Kriegsproduktion, Erlass über Auftragsverlagerung in die besetzten Westgebiete. Vom 3. Dezember 1943.

${ }^{46}$ BArch R 3/470, Anweisung des Rüstungsministers an alle Ausschüsse und Ringe und mit der Produktionssteigerung beauftragten Gruppen, Betr.: Kriegswirtschaftliche Nutzbarmachung der besetzten Westgebiete, 14.12.1943. Für Italien vgl. BArch R 3/393, Speer an die Hauptausschüsse, Hauptringe, mit der Produktionssteuerung beauftragten Gruppen, Betr.: Nutzbarmachung der italienischen Rüstung und Kriegsproduktion, 9.2.1944.

47 BArch R 3/470, Hans Kehrl an verschiedene Stellen, Betr.: Auftragsverlagerung, Preisausgleich bei der Einfuhr ausländischer Waren, 10.2.1944; Reichswirtschaftsminister an Reichsstellen, Betr.: Preisausgleich für Einfuhrwaren, 11.2.1944; Reichswirtschaftsminister an Reichsstellen, Betr.: Verbilligung aus den besetzten Gebieten, 17.9.1943; BArch R 3/3040, Der Beauftragte für den Vierjahresplan an den Reichskommissar für die Preisbildung, 15.9.1943, Bl.81-82. Punktuell wurde bereits vor Ende 1942 bei einigen Rohstoffen und bei Waren, die auf Schwarzmärkten erworben worden waren, ein Preisausgleich durchgeführt, der entweder aus öffentlichen Mitteln oder aus Beiträgen der Wirtschaft finanziert wurde. BArch NS 6/671, Reichswirtschaftsminister an den Reichskommissar für die Preisbildung, 8.8.1942, Bl.79; BArch R 2/19538, Reichsfinanzministerium, Vermerk Dezember 1941. Generell zu Genese des Preisausgleichsverfahrens und seinen Hintergründe, vgl. BArch NS 6/671.

48 BArch R 43 II/333, Reichswirtschaftsminister an alle Reichsstellen, 14.12.1942, Bl. 109.

49 BArch R 3/1367, Reichsminister für Rüstung und Kriegsproduktion, 29.6.1944, 6; BArch R 3/3040, Der Staatssekretär des Beauftragten für den Vierjahresplan an den Reichskommissar für die Preisbildung, 26.3.1943, Bl.81f.; BArch R 3/495, Vermerk betreffend Preisausgleich bei der Einfuhr ausländischer Waren.

50 Vgl. z. B. BArch R 2/ 21716, Der Reichsminister für Rüstung und Kriegsproduktion, Merkblatt über Finanzierungshilfen für die Rüstungswirtschaft, 20.8.1944, 14. 
her hatten die besetzten Gebiete den Verbilligungsbetrag zu tragen, kamen also für die Subventionierung der deutschen Verbraucher auf ${ }^{51}$. Die Clearingverschuldung konnte aber dadurch nur unwesentlich verringert werden. Im Jahr 1943 wurden bei einer Gesamteinfuhr von ca. 3 Mrd. RM aus Frankreich gerade einmal 15,3 Mio. RM für den Preisausgleich aufgewendet ${ }^{52}$.

Bei der militärischen Auftragsverlagerung in die besetzten Gebiete galten die gleichen Herstellungs- und Verwendungsverbote wie im Reich ${ }^{53}$. Auch wurden den Unternehmen, wie im Reich, die Rohstoffe durch die entsprechenden staatlichen Stellen zugeteilt ${ }^{54}$. Ebenso wurde das deutsche Preisrecht angewendet ${ }^{55}$. Grundsätzliche Prämisse der Preisbildung war, dass für marktgängige Produkte die deutschen Preise nicht überschritten und Aufträge zu Festpreisen vergeben werden sollten ${ }^{56}$. Bei nicht marktgängigen Produkten, wie das bei den meisten Gütern der militärischen Auftragsverlagerung der Fall war, sollten Festpreis- und nur in der Anlaufzeit Selbstkostenverträge abgeschlossen werden ${ }^{57}$. Die Genehmigung der Preise für die jeweiligen Aufträge oblag dabei deutschen Preisstellen in den besetzten Gebieten, im Fall der Luftrüstung der Preisstelle des Reichsluftfahrtministeriums in Berlin.

\section{Umfang und Struktur der Rüstungsproduktion in den besetzten Gebieten}

Die Zahl der für die deutsche Wehrwirtschaft arbeitenden Firmen in den in der Frühphase des Krieges besetzten Ländern (ohne Protektorat) stieg zwischen Oktober 1940 und Oktober 1941 rasant an - von 2707 Betrieben im Oktober 1940 auf 6889 ein Jahr später ${ }^{58}$. Bis März 1944 erhöhte sich diese Zahl dann nur noch

51 Vgl. BArch R 3/3040, Der Staatssekretär des Beauftragten für den Vierjahresplan an den Reichskommissar für die Preisbildung, 26.3.1943, Bl. 83; der Beauftragte für den Vierjahresplan an den Reichskommissar für die Preisbildung, 15.9.1943, B1.81-82. Normalerweise wurde das Preisausgleichsverfahren mit deutschen Steuermitteln finanziert. BArch R 3/1626a, Eidesstattliches Gutachten von Kurt Hunscha als Beweismaterial für den Militärgerichtshof IV in Nürnberg, 17.7.1948, Bl.30f.

52 BArch R 3101/32287, Sechster Tätigkeits- und Abschlussbericht der deutschen Waffenstillstands-Delegation für Wirtschaft und des Delegierten der Reichsregierung für Wirtschafts- und Finanzfragen bei der französischen Regierung für die Zeit vom 1. Juli 1943 bis 17. August 1944, Salzburg, 15. Dezember 1944, 58.

53 BArch R 3/3029, Bl. 30.

${ }^{54}$ Vgl. z. B. Umbreit, Der Militärbefehlshaber in Frankreich 266.

${ }^{55}$ Zum deutschen Preisrecht und seiner Anwendung, vgl. Jonas Scherner, Die Logik der Industriepolitik im Dritten Reich. Die Investitionen in die Autarkie- und Rüstungsindustrie und ihre staatliche Förderung (Stuttgart 2008) $37 \mathrm{ff}$.

56 BArch R 3/3029, Bl. $20 \mathrm{ff}$.

57 BArch R 3/1367, Richtlinien für die Preisbildung und Zahlungsabwicklung in den außerdeutschen Gebieten, 29.6.1944.

58 BArch R 3/503. 
vergleichsweise geringfügig, nämlich auf 7637 Unternehmen ${ }^{59}$. Diese Entwicklung lief im allgemeinen in den einzelnen Ländern so ähnlich ab, wie in Tabelle $1 \mathrm{am}$ Beispiel Frankreichs verdeutlicht. Daher kann gesagt werden, dass bereits vor dem Amtsantritt Albert Speers die Grundlage für einen bedeutenden Beitrag des besetzten Auslandes an die deutsche Rüstungswirtschaft geschaffen war. Auch in verbündete Staaten wurden spätestens seit 1941 Rüstungsaufträge verlagert ${ }^{60}$.

Tabelle 1: Zabl der Rüstungsproduzenten im besetzten Frankreich (obne Norddepartments)

Oktober $1940 \quad 375$

Juli $1941 \quad 2600$

Juni 1942

August $1943 \quad 3056$

April $1944 \quad 3302$

Quelle: Ludwig Nestler, Europa unter dem Hakenkreuz. Die faschistische Okkupationspolitik in Frankreich (1940-1944) (Berlin 1990) 71.

Die weitgehende Einbindung der besetzten Gebiete in die deutsche Rüstungswirtschaft vor dem Amtsantritt Speers lässt sich ebenfalls an der Entwicklung des Auftragsbestands nachweisen, der mit der Vorbereitung des Russland-Feldzuges massiv anstieg.

Tabelle 2: Auftragsbestand in den besetzten Gebieten (obne Protektorat) zwischen Oktober 1940 und Oktober 1941 (Mio. RM)

$\begin{array}{ll}\text { Oktober 1940 } & 1852 \\ \text { Januar 1941 } & 1919 \\ \text { April 1941 } & 3317 \\ \text { Juli 1941 } & 4612 \\ \text { Oktober 1941 } & 5180 \\ \text { Quelle: BArch R 3/503. } & \end{array}$

Die tatsächlich in den besetzten Gebieten ausgeführten Rüstungsaufträge stiegen ebenfalls ihrer nominalen Höhe nach, wie Tabelle 3 zeigt, während des Krieges stark an, und zwar - anders als etwa Milward für Frankreich behauptet - bereits vor $1942^{61}$ und insbesondere bevor Speer im Jahr 1943 die Verantwortung für die Auftragsverlagerung übernahm. Vergleichsweise unbedeutend blieben hingegen die Lieferungen befreundeter Staaten. Angaben zur Wehrmachtsverlagerungsproduktion für die meisten besetzten Gebiete wurden vom Wehrwirtschafts- und Rüstungsamt, später vom Rüstungsministerium monatlich in einer speziellen, im Herbst 1940 begonnenen Statistik erfasst und liegen nach Ländern unterteilt fast vollständig vor. Allerdings kam es bereits vor Oktober $1940 \mathrm{zu}$ Verlagerungen in die west- und nordeuropäischen Länder, so dass der in Tabelle 3 für 1940 angege-

59 BA-MA RW 19/1790, Übersicht über die verlagerten Wehrmachtaufträge, Bl.5.

60 Vgl. z.B. György Ránki, The Economics of the Second World War (Wien, Köln, Weimar 1993) 322.

61 Milward, The New Order 82. 
bene Wert nur eine Untergrenze darstellt ${ }^{62}$. In dieser Statistik bestehen zudem zwischen Oktober 1940 und Sommer 1944 im Fall einiger der erfassten Gebiete Lücken von wenigen Monaten, die aber durch Inter- bzw. Extrapolation behoben wurden. Nach dem Sommer 1944 dürfte die Rüstungsgüterproduktion zugunsten des Reichs in den von dieser Statistik erfassten Ländern keine große Rolle mehr gespielt haben, da die meisten Gebiete befreit worden waren, insbesondere die, die in hohem Maß Rüstungsgüter hergestellt hatten (Frankreich, Belgien und das Generalgouvernement). Die Rüstungsproduktion des Protektorats, Italiens und Ungarns für das Reich sind in der erwähnten Statistik zur Wehrmachtsverlagerungsproduktion nicht enthalten. Aus anderen Quellen lässt sich allerdings der jeweilige Produktionswert auch hier, aufgeschlüsselt für die verschiedenen Jahre, ermitteln.

Die Statistiken zur Wehrmachtsverlagerungsproduktion umfassten, wie bereits erwähnt, nach einer Anweisung des Wehrwirtschaft- und Rüstungsamts lediglich solche industriellen Aufträge, die nicht von den Besatzungstruppen vor Ort verbraucht wurden ${ }^{63}$. Kriegsgerät im eigentlichen Sinn und ihre Teile, also Güter, die im deutschen Rüstungsindex erfasst wurden, wie Waffen, Munition, Panzerketten, Tragflächen etc., machten am Wert der verlagerten Wehrmachtsaufträge allerdings nur knapp zwei Drittel aus. Das verbleibende Drittel bestand aus anderen von der Wehrmacht verwendeten industriellen Gütern, wie Maschinen, Bekleidung, „Allgemeines Heeres-Gerät“ (Stahlhelme, Panjewagen, Sanitätsgeräte etc.) oder so genanntes „handelsübliches Gerät“ (Benzinfässer, Glühbirnen, Farbe, Möbel etc.) ${ }^{64}$. $\mathrm{Zu}$ beobachten ist, dass im Zeitablauf die Rangfolge unter den Produktgruppen in den jeweiligen besetzten Gebieten weitgehend unverändert blieb. So hatte die Munitionsfertigung im Generalgouvernement in jedem Jahr einen wertmäßigen Anteil von ca. $30 \%$ an der dortigen Verlagerungsproduktion, während in den Niederlanden z.B. der Schiffbau immer an erster oder an zweiter Stelle stand. In Frankreich wiederum spielte die Kfz- und die Flugzeugproduktion eine wichtige Rolle. Offensichtlich beherzigte man die oben erwähnte Vorgabe des Reichswirtschaftsministeriums aus dem Jahr 1940, Rüstungsaufträge nur an solche Firmen zu verlagern, die bereits Erfahrungen bei der Produktion ähnlicher Güter hatten. Insofern kam es in dieser Hinsicht zu einer durchaus rationalen Ausbeutung. Diese Spezialisierung scheint sich innerhalb der einzelnen Produktkategorien fortge-

\footnotetext{
${ }^{62}$ Vgl. z. B. R 11/88, Zentralauftragsstelle in Belgien und Nordfrankreich, 14.10.1941, Die bisherige Verlagerung von Fertigungsaufträgen nach Belgien und Nordfrankreich, Bl.149.

${ }^{63}$ BArch R 3/503, Rüstungsinspektion Belgien an den Reichsminister für Bewaffnung und Munition, 22. 8.1942, Betr.: Ausnutzung der besetzten Gebiete; BArch R 3/1769, Der Leiter des Planungsamtes an Speer, 31.7.1944, Bl.21. Allerdings ist die Herausrechnung der Reparaturen von Flugzeugen, Schiffen und Kfz, die auch in diesen Werten enthalten sind, nicht möglich. Diese spielten aber keine große Rolle. Vgl. z. B. BArch R 3/1007, Ausnutzung der besetzten Gebiete, Rü. In. Niederlande, Meldemonat August 1942.

${ }^{64}$ Zur Definition dieser Begriffe, vgl. z. B. BArch R 3/1007, Ausnutzung der besetzten Gebiete, Rü. In. Niederlande, Meldemonat August 1942.
} 


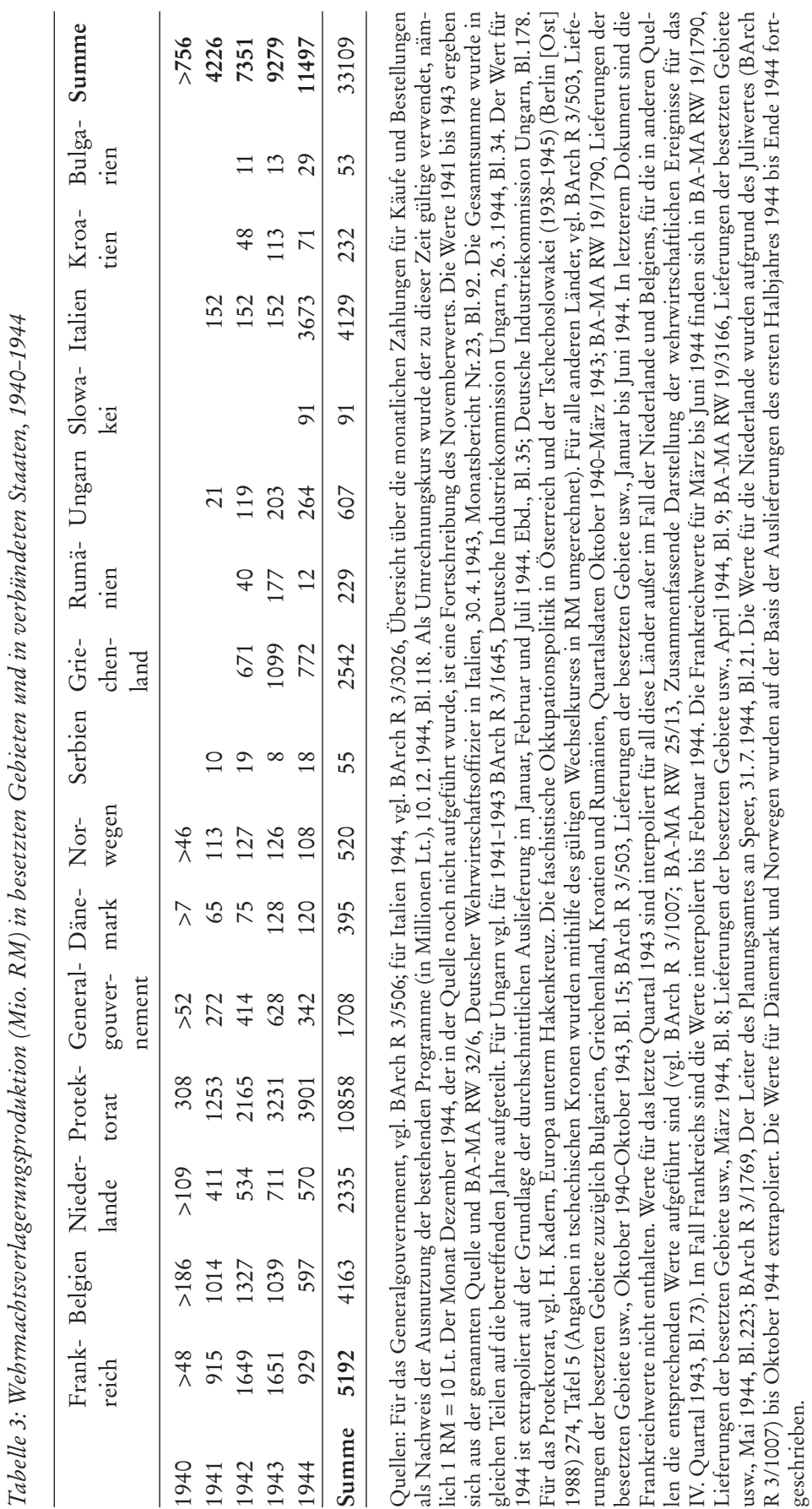


setzt zu haben. So wurden Anfang 1944 47\% der gesamten Panzerkettenfertigung für die Wehrmacht in Frankreich hergestellt ${ }^{65}$.

Überwiegend, wie es immer wieder in den Quellen heißt, wurde bei eigentlichem Kriegsgerät die Teile-, nicht aber die Endfertigung verlagert ${ }^{66}$. Der Verzicht auf Endfertigung ging so weit, dass selbst bei unkomplizierten Rüstungsgütern, wie Munition, Teile gefertigt wurden ${ }^{67}$. Das hatte mit den bereits erwähnten Sicherheitserwägungen, aber auch mit Effizienzgründen zu tun, da häufige Typenwechsel in der Endproduktion den Einsatz neuer Werkzeugmaschinen nach sich zogen und bei Endprodukten ein enger Kontakt zwischen Besteller und Hersteller notwendig war. Außerdem glaubten die deutschen Stellen, wie erwähnt, dass gerade bei der Endfertigung die Motivation der Beschäftigten, für die Besatzer zu produzieren, in den unterworfenen Gebieten besonders gering sei. Dafür aber, dass am ehesten Effizienzgründe für den hohen Anteil der Teileverlagerung ausschlaggebend waren, spricht erstens, dass auch in Bereichen militärischer Auftragsverlagerung außerhalb der eigentlichen Kriegsgerätefertigung, wie nachweislich im Maschinenbau zwischen Juni 1940 und Ende 1942, zu 43\% Teile, nicht aber Endprodukte gefertigt wurden ${ }^{68}$. Zweitens hatte in verbündeten Ländern die Teilefertigung ebenfalls einen hohen Anteil an der Verlagerungsproduktion, wie in Ungarn im Februar 1944 mit mindestens 30\% ${ }^{69}$. Und schließlich ist zu bedenken, dass deutsche Unternehmen, die sich ja in der Frühphase des Krieges oft selbst Verlagerungspartner suchten und später über die Ringe und Ausschüsse bei der Auftragsvergabe beteiligt waren, eher ein Interesse an einer Teileverlagerung gehabt haben dürften. Denn sie gingen davon aus, dass in absehbarer Zeit eine generelle Normalisierung der Wirtschaftspolitik eintreten würde, und damit auch ein Ende der rüstungswirtschaftlich induzierten Auftragsverlagerung ${ }^{70}$. Aus diesem

65 BArch R 3/1769, Rüstungsobmann in Frankreich an Hans Kehrl, 22. 1. 1944, Bl.71.

66 Vgl. z.B. BA-MA RW 19/1332, Wehrwirtschaftliche Folgen einer angenommenen Räumung von Frankreich und Belgien bis zur Reichsgrenze von 1914, B1.311; Feldwirtschaftsamt Inland, Wehrwirtschaftliche Lage bei Fortfall folgender Länder, 1.9.1944, Bl.263-9; BArch N 1340/474, Interrogation vom 21. Juni 1945; Interrogation vom 8. August 1945; BArch R 3/1082, Aktennotiz, Besuch des Herrn Weissenborn - Hauptausschuss Waffen beim Ruk-Stab Mailand und OKH Wa in Como, 3.12.1943; Universität Hohenheim, Nachlass Wagenführ, Band 1, Aktenvermerk, Arbeitsproduktivität. Statistik über Arbeitseinsatz, Fritz Grotius, Kiel, den 8.10.1946; Organisation des maschinellen Berichtswesens des Reichsministers für Rüstung und Kriegsproduktion, S.17.

67 Vgl. z. B. BArch R 3/1007, Ausnutzung der besetzten Gebiete, Rü. In. Niederlande, Meldemonat August 1942.

68 BArch R 3/1404a, Auftragsverlagerung nach Produktionsgruppen, 26.3.1943.

69 BArch R 3/1645, Auftragsbestand Februar 1944, Bl.25. In diesem Dokument ist von direkten und indirekten Aufträgen für die einzelnen Waffengattungen die Rede. Allerdings konnten direkte Lieferungen an eine der Waffengattungen ebenfalls Teile umfassen, wenn z. B. diese an wehrmachtseigene Betriebe geliefert wurden. Neben diesen Lieferungskategorien ist noch vom „gemeinsamen Luftwaffenprogramm“ die Rede. Dieses umfasste aber keineswegs nur die Endfertigung der $\mathrm{He}$ 210 und der Bf 109, sondern zu 20\% des ausgelieferten Wertes auch Motoren-, und damit Teilefertigung. BArch R 3/1645, Deutsche Industriekommission Ungarn, 26.3.1944, Bl.32-3.

70 Generell zur Normalisierungserwartung in der deutschen Industrie während der NS-Zeit, vgl. Scherner, Logik der Industriepolitik im Dritten Reich. 
Grund dürfte es für die Unternehmen naheliegend gewesen sein, möglichst wenig von ihrem Know-how zukünftigen Konkurrenten auf mehr oder weniger liberalisierten Märkten preiszugeben, was natürlich durch eine Verlagerung von Teilen eher als durch die von Endprodukten erreicht werden konnte. Dass derartige langfristige Überlegungen bei der selbstständigen Auftragsverlagerung eine Rolle spielten und dass man zudem mögliche Verlagerungspartner auch unter dem Aspekt langfristiger Kooperationsmöglichkeiten auswählte, zeigt das Beispiel der Robert Bosch $\mathrm{GmbH}$, für die außerhalb der Reichsgrenzen im Jahr 1943 etwa 7000 Beschäftigte in 120 Firmen - etwa Peugeot in Frankreich ${ }^{71}$ - meist Teile produzierten: „Wir haben, soweit kriegswirtschaftliche Erwägungen allein für die Wahl einer bestimmten Fertigungsstätte noch nicht ausreichten, auch Erwägungen, die sich auf die Nachkriegszeit beziehen, berücksichtigt. Bei einem Teil der als verlängerte Werkbänke oder Nachbauer zugezogenen Firmen scheinen die Voraussetzungen für eine dauernde, also über den Kriege hinausgehende Zusammenarbeit gegeben zu sein."72 Auch machte sich die Robert Bosch GmbH in diesem Schreiben Gedanken darüber, dass in manchen Fällen Verlagerungsfirmen, die das Unternehmen technisch beriet, nach dem Krieg zu neuen Wettbewerbern werden könnten; man glaubte allerdings, dass dieses Risiko dadurch aufgewogen werden würde, dass man aufgrund der Verlagerung keine Investitionen tätigen musste und die Arbeitsproduktivität höher war, als wenn man die ausländischen Beschäftigten zwingen würde, im Reich zu arbeiten.

Tabelle 4: Vorleistungsanteil an den Wehrmachtsverlagerungsaufträgen in verschiedenen besetzten Gebieten (\%)

\begin{tabular}{llcc}
\hline Land & Monat/Quartal & $\begin{array}{l}\text { Vorleistungsanteil an der } \\
\text { gesamten Wehrmachts- } \\
\text { verlagerungs-produktion }\end{array}$ & $\begin{array}{l}\text { Vorleistungsanteil an der } \\
\text { eigentlichen Kriegsgerät- } \\
\text { verlagerungs-produktion }\end{array}$ \\
\hline Dänemark & August 1941 & 43 & 53 \\
Generalgouvernement & Dezember 1941 & $>37$ & $>56$ \\
Niederlande & IV. Quartal 1943 & 34 & 40 \\
Niederlande & Februar 1943 & 34 & 41 \\
Niederlande & September 1942 & 46 & 63 \\
\hline
\end{tabular}

Eigene Berechnungen. Quellen: Für Dänemark, vgl. BArch R 3/507, für das Generalgouvernement, vgl. BArch R 3/506. Die Mindestangabe im Fall des Generalgouvernements ergibt sich daraus, dass bei manchen Warenkategorien nicht ersichtlich ist, welchen Anteil die Teilefertigung hatte. Das gilt insbesondere für die Kategorie „Waffen u. Teile“ sowie „Kampfwagen u. Teile“, die zusammen einen Anteil von annährend 10\% an der Gesamtverlagerung des Stichmonats hatten. Aus Vorsichtsgründen wurden die unter diese Kategorien fallenden Aufträge als Endfertigung betrachtet. Für die Niederlande, vgl. BArch R 3/1007.

71 BA-MA RW 20-5/1, Kriegstagebuch der Rüstungsinspektion V, 3. Quartal 1943, Robert Bosch GmbH, 17.7.1943, Betr.: Nutzbarmachung fremder Werkstätten durch Fertigungsverlagerung; BA-MA RW 20-5/2, Kriegstagebuch der Rüstungsinspektion V, 4. Quartal 1943, Robert Bosch GmbH an RüIn V, 29.11.1943, Bl.43ff.

72 BA-MA RW 20-5/1, Kriegstagebuch der Rüstungsinspektion V, 3. Quartal 1943, Robert Bosch $\mathrm{GmbH}$, 17.7.1943, Betr.: Nutzbarmachung fremder Werkstätten durch Fertigungsverlagerung. 
Aufgrund der Tatsache, dass die Kriegsgeräteherstellung zusammen mit der Maschinenproduktion über die gesamte Kriegszeit hinweg etwa einen Anteil von 70 bis $75 \%$ an der militärischen Auftragsverlagerung hatte, und angesichts des Umstandes, dass oft die Produktion von Teilen verlagert wurde, kann man folgern, dass 30 bis $50 \%$ der gesamten Auftragsverlagerung keine Endprodukte betraf, sondern Vorleistungen des metallverarbeitenden Sektors. Diese Größenordnung kann für einige besetzte Länder auch klar bestätigt werden, wie Tabelle 4 zeigt. Für diese Gebiete sind nämlich in manchen Monaten exakte Daten über die Zusammensetzung der Wehrmachtsverlagerungsfertigung überliefert, aus denen sich der Anteil der Teilefertigung genau bestimmen lässt.

\section{Die Bedeutung der verlagerten Wehrmachtaufträge für Deutschlands Rüstung}

Welche Bedeutung hatten die verlagerten Wehrmachtaufträge für die deutsche Rüstungsproduktion? Zur Beantwortung dieser Frage müssen mehrere Probleme gelöst werden. Ein erstes ist, dass sich mit Kriegsbeginn das ohnehin vorhandene Preisgefälle zwischen dem Reich und den besetzten Gebieten, das aus der Überbewertung der Reichsmark seit dem Beginn der 1930er Jahre resultierte, erneut erhöhte, da die Besatzungsmacht die Reichsmark gegenüber den Währungen der besetzten Gebiete weiter aufwertete ${ }^{73}$. Ein zweites Problem ergibt sich daraus, dass in den Ländern, die Wehrmachtsaufträge ausführten, während des Kriegs in unterschiedlicher Intensität Inflationstendenzen auftraten, die sich darin äußerten, dass bei festen Wechselkursen die Kaufkraft der Reichsmark in diesen Gebieten zurückging.

Für die meisten besetzten Gebiete hat die Forschungsstelle für Wehrwirtschaft, eine der Vierjahresplanbehörde angeschlossene Institution, diese Bewertungsprobleme gelöst. Sie ging bei ihren Berechnungen zu Recht davon aus, dass die Wechselkurse vor der Weltwirtschaftskrise letztmalig in etwa die tatsächlichen Kaufkraftparitäten widerspiegelten ${ }^{74}$. Von dieser Basis aus schrieb sie den Kurs in dem Verhältnis fort, in dem sich die Großhandelspreise in Deutschland und den jeweiligen besetzten Gebieten entwickelten, um die tatsächliche Kaufkraft der Reichsmark zu bestimmen ${ }^{75}$. Für Länder, in die Deutschland Aufträge verlagerte und die

73 BArch R 3101/32287, Sechster Tätigkeits- und Abschlussbericht der Deutschen Waffenstillstands-Delegation für Wirtschaft und des Delegierten der Reichsregierung für Wirtschafts- und Finanzfragen bei der Französischen Regierung für die Zeit vom 1. Juli 1943 bis 17. August 1944, Salzburg, 15. 12.1944, S.58; BArch R 2501/15098, Deutsche Reichsbank, Volkswirtschaftliche Abteilung, Stellungnahme zu den Vorschlägen des Auswärtigen Amtes zum Clearingproblem, 15.3.1943, Bl.22f.; John Gillingham, Belgian Business in the Nazi New Order (Gent 1977) 189; Buchheim, Die besetzten Länder.

74 Buchbeim, Die besetzten Länder.

75 Wie erwähnt, kam es seit Februar 1944 zu einem beschränkten Preisausgleich bei Zubehör und Zulieferungen für Kriegsgerät. Allerdings gibt es keinen Hinweis in den Quellen, dass in den hier 
nicht in der Studie der Forschungsstelle für Wehrwirtschaft erfasst sind, wurde grundsätzlich analog vorgegangen, um die Wehrmachtsverlagerungsproduktion in Kaufkraftkursen zu bestimmen ${ }^{76}$. Lediglich Griechenland, wo sich seit 1942 eine Hyperinflation entwickelte, sowie Rumänien, die Slowakei und Kroatien, die nur geringe Beiträge leisteten, und in denen es zum Teil zu erheblicher Inflation kam, sind unberücksichtigt geblieben ${ }^{77}$.

Tabelle 5: Wehrmachtsverlagerungsproduktion der besetzten Gebiete und verbündeten Staaten, 1940-1944 (Mill. RM; Kaufkraftkurse)

\begin{tabular}{|c|c|c|c|c|c|c|c|c|c|c|c|}
\hline & $\begin{array}{l}\text { Frank- } \\
\text { reich }\end{array}$ & Belgien & $\begin{array}{l}\text { Nieder- } \\
\text { lande }\end{array}$ & $\begin{array}{l}\text { Protek- } \\
\text { torat }\end{array}$ & $\begin{array}{l}\text { General- } \\
\text { gouver- } \\
\text { nement }\end{array}$ & $\begin{array}{l}\text { Däne- } \\
\text { mark }\end{array}$ & $\begin{array}{l}\text { Nor- } \\
\text { wegen }\end{array}$ & $\begin{array}{l}\text { Ser- } \\
\text { bien }\end{array}$ & Ungar & Italien & Summe \\
\hline 1940 & $>95$ & $>200$ & $>114$ & 269 & $>26$ & $>7$ & $>52$ & & & & $>763$ \\
\hline 1941 & 1409 & 1090 & 394 & 1031 & 136 & 63 & 116 & 8 & 17 & 320 & 4584 \\
\hline 1942 & 2111 & 1327 & 492 & 1747 & 207 & 69 & 118 & 12 & 79 & 286 & 6448 \\
\hline 1943 & 1651 & 1000 & 645 & 2569 & 314 & 116 & 117 & 3 & 100 & 190 & 6705 \\
\hline 1944 & 929 & 574 & 521 & 3101 & 171 & 110 & 100 & 4 & 96 & 901 & 6507 \\
\hline Summe & 6195 & 3191 & 2166 & 8717 & 1354 & 365 & 503 & 27 & 292 & 1697 & 25007 \\
\hline
\end{tabular}

Zur Methode, vgl. Text. Quellen: Buchheim, Die besetzten Länder; Statistisches Jahrbuch für das Deutsche Reich 1941/42, S. 478; BArch R 3101/33012, Statistisches Reichsamt an Reichswirtschaftsministerium, Betr.: Verrechnungskurse und sonstige intervalutarische Umrechnungssätze des Deutschen Reichs sowie des Auslands, Bl.14-20; B. Mitchell, International Historical Statistics: Europe 1750-1988 (London 1992) 840ff. Bei Dänemark, Norwegen, Serbien, für welche die Forschungsstelle für Wehrwirtschaft die Kaufkraftparitäten nicht in Kalenderjahren, sondern für überlappende Jahre angegeben hat, wurden die Werte entsprechend umgerechnet. Für das Generalgouvernement wurde in Anlehnung an die Forschungsstelle für Wehrwirtschaft unterstellt, dass die Kaufkraft für Rüstungsgüter nur der Hälfte des Wechselkurses entsprach.

Die in Tabelle 5 ermittelten, auf Kaufkraftparitäten beruhenden Werte der Wehrmachtsverlagerungsproduktion müssen nun in einem zweiten Schritt in realen Größen in Preisen von 1943 ausgedrückt werden, um sie zum deutschen Rüstungsindex in Bezug setzen zu können, der ebenfalls in Preisen von 1943 berechnet wurde. Man kann davon ausgehen, dass es bei der ausländischen Fertigung von oft komplexem Kriegsgerät wie im Reich zu Lerneffekten kommen konnte.

verwendeten Verlagerungsstatistiken der Preisausgleich in die RM-Beträge bereits einbezogen worden wäre. Daher wird auch für 1944 die beschriebene Methode angewendet.

${ }^{76}$ Diese Methode impliziert, dass Rüstungsgüter gleicher Beschaffenheit nach Überwindung der Anlaufzeit in Ländern, deren Währung stark unterbewertet war, zunächst grundsätzlich billiger gewesen sein müssen als im Reich, da ja die Verlagerung, wie bereits an anderer Stelle erörtert, im allgemeinen nur an effiziente und erfahrene ausländische Firmen erfolgte. Deren realer Wert, gerechnet in Reichsmark, war demnach anfangs höher als der nominale. Der ehemalige Chef des Wehrwirtschafts- und Rüstungsamtes, der für die Verlagerung zuständig war, spricht jedenfalls auch davon, dass im allgemeinen die Preise für Rüstungsgüter unter denen im Reich gehalten werden konnten. Vgl. Thomas, Geschichte der deutschen Wehr- und Rüstungswirtschaft 140.

77 In diesen Ländern lagen die Preise für Kriegsgerät massiv über den deutschen. Vgl. z. B. BArch R 3/3200, Auszug aus dem Prüfungsbericht über die Firmen: Waggon-, Maschinen- und Brückenbau AG, Slov. Brod., Staatliches Arsenal Sarajevo und Kroatische Berg- und Hüttenwerke AG, Zenica-Sarajevo. 
Das bedeutet, dass die Preisentwicklung von Rüstungsgütern sich im Zeitablauf zunehmend unterproportional zum allgemeinen Preisindex im betreffenden Land bewegt haben dürfte ${ }^{78}$. Und in der Tat lassen sich für Unternehmen aus verschiedenen besetzten Gebieten, für die Preisprüfungs- oder Wirtschaftsprüfungsakten überliefert sind, Lerneffekte nachweisen, die sich bei bestimmten Produkten in ähnlichen Größenordnungen bewegten wie im Reich ${ }^{79}$. Dementsprechend wurde zur Deflationierung der einzig bekannte Preisindex für die Rüstungsproduktion verwendet, nämlich ein Preisindex, den Karl Hettlage, Leiter des Generalreferats Wirtschaft und Finanzen im Rüstungsministerium, das zuständig für die Preisprüfung war, im Sommer 1945 für den United States Strategic Bombing Survey erstellt hat.

Es ist klar, dass die hier vorgeschlagene Methode zur Berechnung der realen Verlagerungsproduktion nur eine grobe Approximation der Wirklichkeit darstellen kann. Angesichts des bruchstückhaft überlieferten Quellenmaterials hinsichtlich des relativen Preisniveaus der Rüstungsgüterproduktion in verschiedenen ausländischen Territorien erscheint aber eine bessere Annäherung an die Realität ausgeschlossen. Der auf diese Weise berechneten realen ausländischen Rüstungsproduktion wird einmal der gesamte reale Industrieabsatz an die Wehrmacht und zum anderen der reale Absatz von Kriegsgerät im engeren Sinn (entsprechend der Klassifikation des vom Rüstungsministerium erstellten Rüstungsindex) gegenübergestellt. Zudem wird der Anteil der Wehrmachtsverlagerungsaufträge an den Einfuhren des Reichs ermittelt, um die Frage zu beantworten, wer Hauptprofiteur des eingangs beschriebenen Importbooms während des Krieges war.

Der gesamte Industrieabsatz an die Wehrmacht ist als die Summe des deutschen Fertigwarenabsatzes der Industrie an die Wehrmacht und der Hälfte der Wehrmachtsverlagerungsproduktion in Kaufkraftkursen berechnet. Damit soll dem

78 Im Fall der deutschen Rüstungsindustrie im Zweiten Weltkrieg, vgl. z.B. Lutz Budraß, Jonas Scherner, Jochen Streb, Fixed-price Contracts, Learning and Outsourcing: Explaining the Continuous Growth of Output and Labour Productivity in the German Aircraft Industry during World War II, in: Economic History Review 63,1 (2010) 107-136; Jonas Scherner, Jochen Streb, Wissenstransfer, Lerneffekte oder Kapazitätsausbau? Die Ursachen des Wachstums der Arbeitsproduktivität in den Werken der Deutschen Sprengchemie GmbH, 1937-1943, in: Zeitschrift für Unternehmensgeschichte 53 (2008) 100-122.

79 Vgl. z.B. BArch R 8135/5470, Kostenprüfung der Deutschen Revisions- und Treuhand AG bei der SECM und der SNCA-SO, 1944. Für weitere Beispiele, siehe BArch R 8135/5587, Prüfungsbericht der Deutschen Revisions- und Treuhand AG über den Jahresabschluß 1941 der Societe des Moteurs Salmson; Prüfungsbericht der Deutschen Revisions- und Treuhand AG über den Jahresabschluß 1942 der Societe des Moteurs Salmson; BArch R 8135/7390, Prüfungsbericht der Deutschen Revisions- und Treuhand AG über den Jahresabschluß des Rumpfgeschäftsjahrs vom 1.1. bis 31.3.1941 der Flugzeugwerke Mielec; Prüfungsbericht der Deutschen Revisionsund Treuhand AG über den Jahresabschluß 1942/43 der Flugzeugwerke Mielec; BArch R 8135/7942, Prüfungsbericht der Deutschen Revisions- und Treuhand AG über den Jahresabschluß 1941 der AG vorm. Skodawerke in Pilsen; BArch R 3/3200, Aktennotiz, Betr.: Preisprüfung bei der Firma Nordböhmische Elektrizitätszähler Tschakowitz bei Prag, 4. 9.1944; Aktennotiz, Betr.: Preisprüfung bei der Fa. Bartos \& Co, Hedwigsthal b. Tschaslau am 31.8. und 31.9.1944. 
Umstand Rechnung getragen werden, dass die andere Hälfte der Wehrmachtsverlagerungsproduktion Vorleistungen umfasste, die an deutsche Produzenten gingen $^{80}$. Diese Vorgehensweise dient dem Ausschluss von Doppelzählungen.

Aus der Berechnung der realen kaufkraftbereinigten ausländischen Rüstungsproduktion folgt, wie in Tabelle 6 gezeigt, dass diese relativ stärker wuchs, bevor die Blitzkriegsphase beendet war, ein Befund, der sich schon aus den "rohen“ nominalen Werten, die auf den offiziellen Wechselkursen beruhen, ergeben hatte. Es zeigt sich weiterhin, dass bereits vor Speers Amtsantritt die besetzten Gebiete einen bedeutenden Anteil an der deutschen Rüstungsproduktion innehatten. Die vom Rüstungsministerium seit 1943 angestrebte Ausweitung der Verlagerung bewirkte im ersten Halbjahr des darauf folgenden Jahres, also bevor sich das für Deutschland produzierende ausländische Gebiet aufgrund des Vorrückens der Alliierten stark verkleinerte, zwar real einen Anstieg gegenüber der durchschnittlichen halbjährlichen Produktion des Vorjahres. Allerdings ist dieser Zuwachs hauptsächlich auf die Einbeziehung Italiens in die deutsche Rüstungsproduktion zurückzuführen. Da aber die gesamte Rüstungsherstellung für die Wehrmacht in Deutschland fast in gleichem Umfang wie die Verlagerungsproduktion wuchs, stieg der relative Beitrag des Auslandes im ersten Halbjahr 1944 gegenüber dem Vorjahr nur unwesentlich an.

Es ist weiterhin festzustellen, dass sich die Relation der eigentlichen Kriegsgeräteproduktion und der industriellen Produktion für die Wehrmacht im Reich und im Ausland offensichtlich nicht stark unterschied; die Anteile der ausländischen Produktion an der jeweiligen Kategorie waren während der gesamten Dauer des Krieges ähnlich. Und schließlich kann man sehen, dass der Anteil der industriellen Wehrmachtsverlagerungsproduktion an der deutschen Einfuhr im Kriegsverlauf massiv anstieg. Berücksichtigt man zudem, dass ja wohl auch ein beträchtlicher Teil des Nahrungsmittel-, Rohstoff- und Halbwarenimports des Reichs mittel- oder unmittelbar für die Wehrmacht bestimmt war, so zeigt sich, dass die Ausbeutung der besetzten Gebiete nur in geringem Umfang den deutschen Konsumenten zugute gekommen sein konnte.

Eine Analyse der realen Werte der Rüstungsproduktion der einzelnen Länder zeigt, dass mit Ausnahme Italiens die westeuropäische Produktion ab 1942/43 stagnierte, während sie in den beiden osteuropäischen Staaten und im Reich weiter anstieg. Dafür gab es mehrere Gründe ${ }^{81}$ : Erstens traf die 1942 einsetzende, im weiteren Verlauf sich verschärfende Zwangsrekrutierung von Arbeitskräften in

${ }^{80}$ Es wurde also die Obergrenze der anteiligen Vorleistungsfertigung an der Verlagerungsproduktion unterstellt. Nimmt man die Untergrenze an, so sind die in Tabelle 6, Spalte II, berechneten Werte geringfügig höher. Nicht berücksichtigt bei dieser Berechnung wurde der deutsche Export von Vorerzeugnissen in diese Länder, da dieser nach den verfügbaren Informationen nicht sonderlich ins Gewicht fiel.

${ }^{81}$ Vgl. BArch R 3/1404a, Die Auftragsverlagerung im Lenkungsbereich Maschinenbau seit Juni 1940, 4. Bericht, S.3. 
Tabelle 6: Europas Beitrag zur Befriedigung der industriellen Webrmachtsnachfrage 1940 bis 1944

\begin{tabular}{|c|c|c|c|c|}
\hline & $\begin{array}{l}\text { (I) } \\
\text { Wehrmachtverlage- } \\
\text { rungsproduktion } \\
\text { des Auslands (in } \\
\text { Preisen von 1943; } \\
\text { Mio. RM) }\end{array}$ & $\begin{array}{l}\text { (II) } \\
\text { Wehrmachtverlagerungs- } \\
\text { produktion des Auslands } \\
\text { in Prozent des gesamten } \\
\text { industriellen Absatzes an } \\
\text { die Wehrmacht }{ }^{3}\end{array}$ & $\begin{array}{l}\text { (III) } \\
\text { Anteil der } \\
\text { ausländischen } \\
\text { Kriegsgeräte- } \\
\text { produktion }{ }^{4} \text { am } \\
\text { Rüstungsindex }\end{array}$ & $\begin{array}{l}\text { (IV) } \\
\text { Wehrmachtver- } \\
\text { lagerungsproduk- } \\
\text { tion }{ }^{6} \text { in Prozent } \\
\text { der tatsächlichen } \\
\text { Importe }\end{array}$ \\
\hline 1940 & $>908$ & $>5$ & $>5,2$ & $>9$ \\
\hline 1941 & 5042 & 21 & 28 & 33 \\
\hline 1942 & 5997 & 25 & 24 & 37 \\
\hline 1943 & 6705 & 21 & 17 & 39 \\
\hline 1944 & 8264 & $(23)^{5}$ & $17(19)^{5}$ & 53 \\
\hline
\end{tabular}

1) Kaufkraftkurswerte aus Tabelle 5 deflationiert mit dem Hettlage-Preisindex.

2) Kaufkraftkurswerte aus Tabelle 5 .

3) Der gesamte industrielle Absatz an die Wehrmacht ist definiert als der direkte deutsche Absatz an die Wehrmacht zuzüglich 50\% der ausländischen Rüstungsproduktion.

4) Nur der Anteil an der ausländischen Rüstungsproduktion gemäß Tabelle 5, die der Güterklassifikation des deutschen Rüstungsindex entspricht. Vgl. dazu und zu den Prozentsätzen Abschnitt 3. Diese Werte wurden mit dem Hettlage-Preisindex deflationiert.

5) Der eingeklammerte Wert für das Jahr 1944 in Spalte (III) stellt den Anteil im ersten Halbjahr 1944 dar.

6) Vgl. Tab. 3 letzte Spalte.

Eigene Berechnung. Zur Methode, vgl. Text. Quellen: Für den direkten Absatz der deutschen Industrie an die Wehrmacht, vgl. National Archives at College Park (im Folgenden NARA) RG 243 (Records of the United States Strategic Bombing Survey), European Survey, Box No 12, 134a, Special Paper No 8, Industrial Sales, Output, and Productivity. Prewar Area of Germany, 1939-44, March 15, 1946, S. 15; für den Hettlage-Preisindex, vgl. NARA RG 243, United States Strategic Bombing Survey, Overall Economic Effects Division, Special Paper No 1, The Gross National Product of Germany 1936-1944, S. 5-9; für den Wert der im Rüstungsindex erfassten Güter, ausgedrückt in Preisen von 1943, vgl. Jonas Scherner, Jochen Streb, Armament Production in the Third Reich (unveröffentlichtes Manuskript, 2008) sowie Rolf Wagenführ, Die deutsche Industrie im Kriege 1939-1945 (Berlin 1954) S.73, 114; für die Importe vgl. Scherner, Der deutsche Importboom; für die Rüstungsproduktion des Auslands (in laufenden Preisen), vgl. Tabelle 3 und Tabelle 5 (Kaufkraftwerte).

besonders starkem Maß die Industrie der westeuropäischen Länder ${ }^{82}$. Im Generalgouvernement und im Protektorat standen die Rüstungsproduzenten hingegen unter der Kontrolle deutscher Firmen bzw. des Reichs ${ }^{83}$, so dass sie vor einer Zwangsrekrutierung ihrer Arbeitskräfte geschützt waren. Seit 1942 stieg die Zahl der Beschäftigten in der Rüstungsindustrie im Protektorat sogar schneller als im Reich $^{84}$. Möglicherweise spielten die faktischen Eigentumsverhältnisse auch insofern eine Rolle, als sich in diesen Gebieten die Rüstungskapazitäten durch deutsche Investitionen, die direkt oder indirekt vom Staat finanziert wurden, deutlich ausweiteten $^{85}$, während von entsprechenden Vorgängen in den westlichen besetz-

82 Vgl. z.B. BA-MA RW 25/8, Lagebericht der Rüstungsinspektion Belgien, 1.10.1942, Bl.95; Umbreit, Der Militärbefehlshaber in Frankreich 270; Ránki, The Economics of the Second World War 334.

${ }^{83}$ Vgl. etwa Hopmann, Von der MONTAN zur Industrieverwaltungsgesellschaft $287 \mathrm{f}$.

${ }^{84}$ Vgl. BA-MA RW 46/492, Bl.118f.

85 Vgl. z.B. Overy, War and Economy 339 Tab.10.1. 
ten Gebieten nichts Nennenswertes bekannt ist. Auch werden immer wieder der sich verschärfende Rohstoff- und Energiemangel, Transportschwierigkeiten und die sich im Zuge der militärischen Rückschläge Deutschlands verstärkenden Sabotageakte als eine Barriere für eine weitere Ausweitung der Produktion in den westlichen Gebieten angeführt ${ }^{86}$. Außerdem tätigten die deutschen Behörden seit 1942 in hohem Umfang Käufe auf den französischen, belgischen und niederländischen Schwarzmärkten. Das führte - wie immer wieder in den Quellen hervorgehoben wird - zu einem nachlassenden Interesse der Unternehmen an der regulären und damit preisüberwachten Auftragsverlagerung, zumindest bis Frühjahr 1943, als die „offiziellen“, also durch Behörden getätigten Schwarzmarktkäufe u. a. wegen dieser Anreizproblematik prinzipiell eingestellt wurden ${ }^{87}$.

Schließlich kam hinzu, dass sowohl das Generalgouvernement als auch das Protektorat im Vergleich zu den westlichen besetzten Ländern als verhältnismäßig luftsicher galten und auch deshalb seitens deutscher Stellen eine verstärkte Produktion dort angestrebt wurde ${ }^{88}$. Im Fall von Belgien wurde die Verlagerung der Rüstungsproduktion noch dazu dadurch erschwert, dass es seit 1942 zu zunehmendem Widerstand der belgischen Behörden gegen die Rüstungsproduktion zugunsten des Besatzers kam, verstieß diese doch nicht nur gegen das Völkerrecht, sondern war durch das belgische Strafgesetzbuch ebenfalls verboten ${ }^{89}$. Die deutschen Besatzer hatten aber kein Interesse, sich zu offensichtlich über diese Widerstände hinwegzusetzen. Denn Rüstungsgüter, soweit sie im Clearingverkehr finanziert wurden, sollten nach ausdrücklicher Anweisung deutscher Behörden bei den entsprechenden Devisenanträgen getarnt sein, so dass den belgischen Clearingstellen der wahre Charakter der in Belgien für das Reich produzierten Güter und insbesondere der Umfang dieser Produktion möglichst verborgen blieb. Trotzdem kann aus dieser Entwicklung keineswegs geschlossen werden, dass die Bedeutung der westlichen Länder für die deutsche Kriegswirtschaft generell zurück ging. Zwar war seit 1942 der Anteil der Rüstungsproduktion an den Aus-

86 Vgl. z. B. Umbreit, Militärbefehlshaber in Frankreich 270; BArch R 3/1404 a, Die Auftragsverlagerung im Lenkungsbereich Maschinenbau seit Juni 1940, 4. Bericht, S. 3; BArch R 3101/32287, Sechster Tätigkeits- und Abschlussbericht der deutschen Waffenstillstands-Delegation für Wirtschaft und des Delegierten der Reichsregierung für Wirtschafts- und Finanzfragen bei der französischen Regierung für die Zeit vom 1. Juli 1943 bis 17. August 1944, Salzburg, 15. Dezember 1944, B1.7.

87 Vgl. z.B. BA-MA RW 35/824, Abschlussbericht der ZAST Belgien/Nordfrankreich, S. 55. Allerdings fanden auch noch danach „offizielle“ Schwarzmarktkäufe statt, wenn auch der Umfang zurückgegangen zu sein scheint. Für ein Beispiel, vgl. BArch R 2 Anh./79, Aktenvermerk, Der Reichsminister für Finanzen, 30.9.1943, Bereitstellung von ffrs aus Besatzungskostenmitteln für Ankäufe der Firma Pimetex, B1.83.

88 BArch R 3/1626a, Eidesstattliches Gutachten von Kurt Hunscha als Beweismaterial für den Militärgerichtshof IV in Nürnberg, 17.7.1948, Bl.2.

89 BA-MA RW 25/13, Zusammenfassende Darstellung der wehrwirtschaftlichen Ereignisse für das 4. Quartal 1943, Bl.67; BArch R 8 IX/19, ZAST in Belgien und Nordfrankreich, Januar 1944. 
fuhren dieser Länder in das Reich rückläufig, aber es ist weiterhin zu realen Exportzuwächsen gekommen ${ }^{90}$.

Insgesamt bleibt festzuhalten, dass das Protektorat für die deutsche Rüstungsproduktion das wichtigste Land war und 1943/44 tatsächlich in etwa den Anteil erreichte, den die deutschen Behörden bereits vor seiner Schaffung prognostiziert hatten ${ }^{91}$. Zwar war die Rüstungsproduktion für die Wehrmacht zu Beginn des Kriegs noch gar nicht so hoch. Das lag aber daran, dass man das dortige Rüstungspotential zunächst zur Beschaffung von Devisen ausnutzte und noch Aufträge für spätere Feindstaaten, wie die Sowjetunion und Jugoslawien, ausgeführt wurden ${ }^{92}$.

Den beträchtlichen deutschen Rüstungsimporten standen nur relativ geringe deutsche Ausfuhren von Kriegsgerät gegenüber, die seit 1941 überwiegend auf die verbündeten Staaten Rumänien, Italien und Bulgarien entfielen ${ }^{93}$. Für eine Gegenüberstellung der deutschen Rüstungsexporte und der realen, in RM ausgedrückten Rüstungsimporte muss allerdings berücksichtigt werden, dass Deutschland für seine Kriegsgeräteausfuhren überhöhte Preise verlangte ${ }^{94}$. Im Jahr 1943, für das exakte Angaben überliefert sind, machte die Verteuerung in etwa $100 \%$ des Inlandspreises aus ${ }^{95}$. Sie ist darauf zurückzuführen, dass die Reichsmark gegenüber den Währungen dieser Länder zunehmend unterbewertet war, man sich aus politischen Gründen aber auf deutscher Seite scheute, Druck zur Anpassung der Paritäten auszuüben ${ }^{96}$. Deshalb ging man von deutscher Seite seit Mitte des Krieges eben dazu über, die Preise deutscher Ausfuhrwaren den gestiegenen Preisen für Einfuhren aus diesen Ländern anzupassen ${ }^{97}$. Im Fall Rumäniens war bereits im so genannten Ölpakt festgelegt worden, dass die Preise deutscher Rüstungsexporte in gleichem Maß steigen sollten wie der Preis rumänischer Erdölexporte nach Deutschland, der sich zwischen 1940 und 1943 insgesamt um 157\% erhöhte ${ }^{98}$.

Wenn auch in anderen Jahren die deutschen Kriegsgeräteexporte in einem ähnlichen Ausmaß überteuert waren, wie für 1943 nachgewiesen, so ergibt sich, dass

\footnotetext{
90 Scherner, Der deutsche Importboom.

${ }^{91}$ Für die Prognose vgl. BA-MA, RW 22/21, Geschichte der Rüstungsinspektionen, Bl. 5 .

92 Vgl. z.B. BA-MA RW 46/2; BArch R 8135/7942, Prüfungsbericht der Deutschen Revisionsund Treuhand AG über den Jahresabschluß 1941 der AG vorm. Skodawerke in Pilsen.

93 BArch R 901/68585, Kriegsgerätabschlüsse in den Jahren 1939 bis 1943 nach Ländern und Ländergruppen, Bl.128-131.

${ }_{94}$ Für die entsprechenden Vorschriften, vgl. BArch R 3/545.

95 BArch R 3/1959, Kriegsgeräteausfuhr 1943, Bl.54-56.

${ }^{96}$ Vgl. z. B. BArch NS 6/671, Der Reichskommissar für die Preisbildung an die Partei-Kanzlei, 27. 4.1942, Bl.3; Andreas Hillgruber, Hitler, König Carol und Marschall Antonescu. Die deutschrumänischen Beziehungen 1938-1944 (Wiesbaden 1965) 205.

97 BArch R 3/1626a, Eidesstattliches Gutachten von Kurt Hunscha als Beweismaterial für den Militärgerichtshof IV in Nürnberg, 17.7.1948, Bl.30.

98 BArch R 3101/32275, Gesandter Carl Clodius an General Debre, Königlich Rumänischer Rüstungs- und Wirtschaftsminister, 11.8.1944, Bl.12; BArch R 3102/3551, Forschungsstelle für Wehrwirtschaft, Belastung der deutschen Währung durch überhöhte Auslandspreise und Clearingschulden, 3.11.1942, Bl.6f.
} 
der Kriegsgeräteexport Deutschlands zwischen 1940 und 1943, in inländischen Preisen betrachtet, sich auf etwa 1,7 Mrd. RM und real, in Preisen von 1943, auf ca. 1,8 Mrd. RM belief ${ }^{99}$. Dieser Betrag ist aber nicht gleichzusetzen mit der realen deutschen Rüstungsfertigung für das Ausland. Nur etwa 50\% der Exporte erfolgte aus direkten Lieferungen deutscher Rüstungsfirmen, ca. 25\% aus Wehrmachts-, der Rest aber aus Beutebeständen.

\section{Ergebnisse}

Es scheint, dass der wirtschaftliche Beitrag Europas für die unmittelbare Kriegsführung Deutschlands im Zweiten Weltkrieg mindestens dem militärischen entsprach. Denn erstens rüsteten sich die verbündeten Truppen offensichtlich in großem Maß selbst aus, da die deutschen Rüstungsexporte relativ unbedeutend blieben. Zweitens produzierten in der Mitte des Krieges die besetzten Gebiete mindestens ein Viertel der gesamten, für die Wehrmacht bestimmten Waffen und Ausrüstungsgegenstände. Auch in diesem Bereich profitierte Speer von der Basis, die in den Jahren zuvor geschaffen worden war. Offensichtlich hat das so oft geschmähte, angeblich „ineffizient“ agierende Wehrwirtschafts- und Rüstungsamt die Grundlagen dafür gelegt, dass Deutschland die Niederlage so lange hinauszögern konnte. Von Kriegsbeginn an spannte Deutschland Unternehmen in den besetzten Gebieten systematisch und nach Effizienzgesichtspunkten für die Rüstungsproduktion ein - ein klarer Beleg gegen die Blitzkriegshypothese. Gegen diese spricht ebenfalls, dass von einer neuen Dimension der realisierten Ausbeutung nach 1942, also nachdem das Rüstungsministerium für die besetzten Gebiete zuständig geworden war, nicht die Rede sein kann. Auch der von Speer Ende 1943 eingeleitete Versuch, die militärische Auftragsverlagerung in die besetzten Westgebieten zu forcieren, führte zu keiner bemerkenswerten realen Steigerung, wahrscheinlich besonders weil es unterlassen worden war, in den westlichen Besatzungsgebieten hinreichend in die entsprechenden Unternehmen $\mathrm{zu}$ investieren. Angesichts des Umstandes massiver Rüstungsinvestitionen im Reich und in Anbetracht der Tatsache, dass die deutschen Kapazitäten im allgemeinen nicht voll ausgelastet waren, entbehrt jedoch der Verzicht auf Investitionen in diesen Ländern, die im Unterschied zum Protektorat und zum Generalgouvernement nicht faktisch zum Reich gehörten und wesentlich luftgefährdeter waren, nicht einer gewissen Logik.

Ob man die vom Reich verfolgte Ausbeutungskonzeption als „optimal“ bezeichnen kann, bleibt allerdings deshalb fragwürdig, weil es in den einzelnen Ländern offensichtlich zu keiner Ausbeutungspolitik „aus einem Guss“ kam, was sich z. B an der bekannten Kontroverse zwischen Sauckel und Speer über die Zwangs-

99 Für den Export (einschließlich Wehrmachtsabgaben, allerdings ohne direkte Frontabgaben) vgl. BArch R 901/68585, Geschäftsberichte der Ausfuhrgemeinschaft für Kriegsgerät, 1940/41, 1941/42, 1942/43, 1943/44, B1.75-126. 
rekrutierung von Arbeitskräften zeigt. Kontraproduktiv waren auch die durch die Ausbeutungspolitik letztendlich verursachten Inflationstendenzen in manchen Ländern ${ }^{100}$. Sie führten nicht nur dazu, dass Deutschland mehr für seine Importe zahlen musste, sondern verursachten wohl auch - jedenfalls bei beschleunigter Inflation - reale Produktivitätseinbußen. Denn rasche Geldentwertung verringerte die Leistungsanreize in den besetzten Ländern ${ }^{101}$. Kontraproduktiv für eine Erhöhung der militärischen Auftragsverlagerung waren auch die „offiziellen“ Schwarzmarktkäufe in den besetzten Westgebieten, da sie es für viele Unternehmen lukrativer machten, Güter für den Schwarzmarkt anstelle für den preisüberwachten Wehrmachtssektor zu produzieren.

\section{Summary}

This paper examines how industrial orders placed by the Wehrmacht throughout Europe contributed to the German war effort. For the first time the focus is not only on the administrative way these orders were placed, but also their quantitative volume in the respective countries, especially relative to the total German war effort. It can be shown that Europe's contribution to the munitions the Wehrmacht needed was significant, approximately matching the share foreign troops had on the Eastern front. At least one quarter of the weapons and equipment used by the Germans was produced abroad. Moreover, it can be demonstrated that Albert Speer, appointed armaments minister at the beginning of 1942, also benefitted from previous achievements in this respect: from the start of World War II onwards, Nazi Germany systematically expanded the proportion of occupied Europe's industrial base that was used for German industrial orders. This research outcome provides further evidence against the Blitzkrieg-Hypothesis.

100 BArch R 2501/15098, Deutsche Reichsbank, Volkswirtschaftliche Abteilung, Stellungnahme zu den Vorschlägen des Auswärtigen Amtes zum Clearingproblem, 15.3.1943, Bl.22f. Die Ursachen der Inflation, etwa die von Deutschland erzwungene Vergabe großer Clearingkredite, war den deutschen Stellen durchaus bewusst. Vgl. z. B. PA AA R 106483, Protokoll über die erste ordentliche Sitzung des Verwaltungsrats der DVK im Jahr 1943, 7.1.1943.

101 Darüber waren sich die deutschen Stellen durchaus im Klaren. BArch R 2/13502, Bericht über die Sitzung des Verwaltungsrats der Reichskreditkassen am 5. Oktober 1942, Bl. 89. 